\title{
Methodology for the identification of alternative manufacturing changes for safety-critical components
}

\author{
Thomas Bergs ${ }^{1} \cdot$ Lennard Hermann $^{1}$. Jan Rey ${ }^{1} \cdot$ Sebastian Barth $^{1}$
}

Received: 15 January 2020 / Accepted: 30 March 2020 / Published online: 3 April 2020

(c) The Author(s) 2020

\begin{abstract}
A variety of factors are forcing companies to change their products and the components these products are composed of. Examples of current factors are changing customer requirements, product optimizations or ever-shorter product lifecycles. These component changes, particularly in safety-critical components in the medical and aerospace industry, pose great challenges and are limited in scope. The challenges and limitations arise from the high costs and the amount of time involved in component recertification as required by law, as well as revalidations of the manufacturing after a change has been implemented. Some component changes are even mandatory. In this case there are no options for reducing recertification efforts by choosing between different design changes. In contrast, there are alternative manufacturing options with which to implement the prescribed component changes that can reduce the amount of revalidation required on the manufacturing side. Due to the fact that changes to the manufacture of safety-critical components require validation, the existence of alternative manufacturing changes offers the potential to save time and money by selecting the best-suited alternative. In order to be able to select the best-suited alternative, all alternative manufacturing changes must be known, which poses a challenge for technology planners dealing with the complex manufacture of safety-critical components. Therefore, a methodology is introduced to support technology planners in identify alternative manufacturing changes with which to implement scope-restricted changes to safety-critical components. The methodology is validated by applying it to a case study from the medical industry.
\end{abstract}

Keywords Technology planning $\cdot$ Manufacturing change management $\cdot$ Aerospace $\cdot$ Medical industry $\cdot$ Manufacturing adaptation $\cdot$ Safety-critical

\section{Introduction}

Ongoing developments such as ever-shorter product lifecycles [1], the dynamization of market needs and product optimization demands [2] are forcing today's manufacturing companies to redesign their products, thereby changing the components that comprise the products ever more frequently. Component changes are not only an issue during the product development phase but also after market entry [3]. In order to implement a component change after market entry, the existing manufacture must also be changed. In the following, changes to an established manufacture

Lennard Hermann

L.Hermann@wzl.rwth-aachen.de

https://www.wzl.rwth-aachen.de

1 Laboratory for Machine Tools and Production Engineering (WZL) of RWTH Aachen University, Campus-Boulevard 30, 52074 Aachen, Germany are referred to as manufacturing changes (MCs) (cf [4].). Manufacturing changes for the implementation of component changes after market entry pose a particular challenge when manufacturing safety-critical components for the medical technology and aerospace industries. This challenge arises from the validation processes which companies are legally required to perform after changing the manufacture of medical technology [5] and aerospace [6] components. This validation currently poses a challenge, particularly for medical products, since a new and more strict Medical Device Regulation (cf [7]) will replace the current Medical Device Directive (cf [8]). Within the new regulation, more products are categorized in highrisk categories, increasing the effort required for validation after changing their manufacturing processes. Since the required validation effort varies from $\mathrm{MC}$ to $\mathrm{MC}$, it becomes an additional criterion to take into account when selecting an MC for a given component change. However, before the most suitable MC can be selected, all alternative 
MCs must be identified in order to form the correct basis for decision making [9]. The manufacture of safety-critical components is usually characterized by a high degree of complexity and a multitude of interdependencies between the components and the manufacturing processes. The systematic identification of alternative MCs for safety-critical components therefore poses a scientific challenge, which is addressed by the methodology introduced here.

The economic relevance of this research lies in the high cost and time efforts of recertification and revalidation processes. E.g., changing the geometry of a cutting tool for turning an aero engine component could cost around $€ 135 \mathrm{k}$ just for the revalidation [10]. The design and redesign [11] as well as requirements [12] and certifications [13] of medical products have already been addressed extensively by researchers. However, changes in the manufacturing of medical products have not yet been adequately discussed. Identifying an alternative Manufacturing Change, capable of implementing the required component change (e.g. adjusting process parameters such as cutting speed or feed rate), which may already be included in the existing certification, can drastically reduce revalidation costs for the manufacture. In the case of medical technology, the systematic identification of alternative MCs represents economic optimization potential. Changes to the manufacture must be presented to a legal entity, which decides to what extent revalidation is requiredfrom a small change in the documentation to revalidation of the entire process chain. Therefore, by considering the relevant aspects of revalidation while identifying alternative MCs has the potential to keep revalidation effort to a minimum.

The reference frame for the conducted research and the application of the developed methodology is defined by process chains and components. Process chains are referred to as sequences of directly value-adding manufacturing processes carried out on defined means of production [14]. Therefore, manufacturing changes describe adaptations to manufacturing processes such as a change in parameters or tools as well as modifications to process chains such as substitute processes or extended process chains. Component changes are referred to as quantitative changes of component characteristics, e.g. roughness, length, radius or evenness.

In the following, the state of research regarding the identification of manufacturing changes is presented and the research gap is identified. Later, the methodology for the identification of manufacturing changes for safety-critical components is presented and validated by applying it to a case study from medical technology, according to the regulations [15]. In conclusion, a summary of the presented research findings and an outlook on future research activities are given.

\section{State of research regarding the identification of alternative manufacturing changes}

To capture the state of research for the identification of alternative manufacturing changes for safety-critical components, the research fields Technology Planning und Manufacturing Change Management (MCM) were examined. Technology Planning deals with the analysis of a component, identification of sufficient manufacturing processes and the generation of process chains to manufacture the component ( $\mathrm{cf}[16]$ ). Manufacturing Change Management originates from the more established Engineering Change Management and focuses on the planning of alterations to a factory [4]. Since a variety of publications exists in both fields, only the most relevant ones will be mentioned subsequently. However, the conclusion drawn from the literature review at the end of this also takes approaches into account that are not mentioned explicitly. The current state of research was examined with regard to three criteria. The first criterion is the reference frame. Since the aim of the presented methodology is to identify MCs for changes of component characteristics such as roughness, diameter or evenness, the reference frame must include components and their characteristics. In addition, the reference frame must cover process chains and the manufacturing processes they are composed of, since a component change can be implemented by adapting different processes or combinations. The second criterion is the existence of a systematic procedure to identify alternative MCs, since the whole procedure must be documented extensively and coherently to present it to a legalized agency for revalidation. The third criterion was the consideration of validation effort involved when implementing a component change.

Within Technology Planning, Klocke et al. developed a methodology for the integrative design of production process and inspection sequences for medical devices. The methodology's reference frame covers component characteristics and process chains, and can be used to identify MCs by generating new process chains for the changed component and comparing them with the existing process chain. Although this represents a systematic procedure, the effort involved in generating completely new process chains to identify alternative MCs is excessively high. Validation efforts are not taken into account [17]. Similar approaches with a different focus but the same deficits for the identification of alternative MCs for safety-critical components were developed by Müller [18] and Schindler [19]. Another methodology taking into account the existence of an established process chain was developed by Denkena et al. The methodology supports the integrative 
production planning process in highly dynamic environments and considers components as well as process chains within its reference frame [20]. However, the methodology does not describe a systematic procedure for identifying alternative MCs, and does not cover the validation effort. Additionally, the methodology's focus lies on flexibility, which is not a dominant target dimension for the manufacture of safety-critical components. Another approach featuring the same deficits was developed by Jacob et al., focusing on the parallel planning of product design and process optimization [21]. Comprehensively, Technology Planning approaches can generally be used to identify MCs and often cover the intended reference frame. However, the effort required to apply these approaches to the identification of alternative MCs is disproportionate due to their different objectives and the lack of consideration of validation. Therefore, they cannot be applied to solve the stated problem in an efficient manner (see Sect. 1), but they do serve as a basis for the development of the approach presented here.

In the field of Manufacturing Change Management, Koch developed an MCM context model [4] and an MCM reference process [22], which enable scientists to locate their research within MCM. Since the context model's objective is to provide a framework, its level of detail is too low for the identification of MCs. A more detailed approach was developed by Malak and Aurich, who presented a methodology for planning technical changes in production systems [23]. The methodology includes a systematic procedure to identify MCs, but with the reference frame of entire factories. Therefore, the detailed consideration of component characteristics and manufacturing processes is not part of the methodology. Validation efforts are not considered. Approaches with similar attributes were developed by Plehn [24] and Cichos et al. [25]. An approach considering component characteristics and offering a systematic procedure to identify MCs was developed by Karl et al. [26]. However, the approach is only applicable to single manufacturing processes, not to process chains, and does not consider validation. Another approach to identifying MCs for single manufacturing processes was developed by Hoang et al. [27]. Marks et al. extended this approach by an agent-based decision support system [28]. Conclusively, approaches from MCM do provide frameworks for a systematic procedure to identify alternative MCs. However, they do not consider validation and either cover too unspecific reference frames (whole factories) or the reference frame is too limited (single manufacturing process) in order to identify alternative adaptations of process chains for implementing changes to a safety-critical component's characteristics.

In addition to the approaches discussed above, further research was carried out into the certification of medical product designs from an engineering perspective (e.g.
[29]). Particularly the Quality by Design (QbD, cf [30]) approach has become a subject of increasing interest for the design and manufacture of medical products [31]. Although these approaches represent major contributions for the design and manufacture of safety-critical components as addressed in this publication, they cannot be used to identify cost-optimized manufacturing changes in order to implement the required component change. They are applied in the earlier phase of component redesign, representing the input for the methodology introduced here. An overview of the problem statement and the presented solution approach is given in Fig. 1.

Bearing these research deficits in mind, the objective of the research conducted here was to develop a methodology for the systematic identification of alternative MCs for safety-critical components, that takes validation into account. The reference frame included process chains and components characteristics. The potential of the methodology introduced here to innovate is represented by the reduction of costs and time required to identify MCs and to recertify the changed manufacturing processes. Therefore, a systematic procedure that integrates recertification-relevant aspects into the process of identifying MCs is provided for the first time. The methodology enables technology planners to systematically identify a costoptimized solution for implementing a required change to a safety-critical component. The intention of the developed methodology was firstly introduced in [9] as part of a broader concept for the general management of manufacturing changes for safety-critical components. However, no details about the identification of MCs were given in the concept and further research was required, hindering its application and making it necessary to thoroughly explain the identification of MCs within this publication.

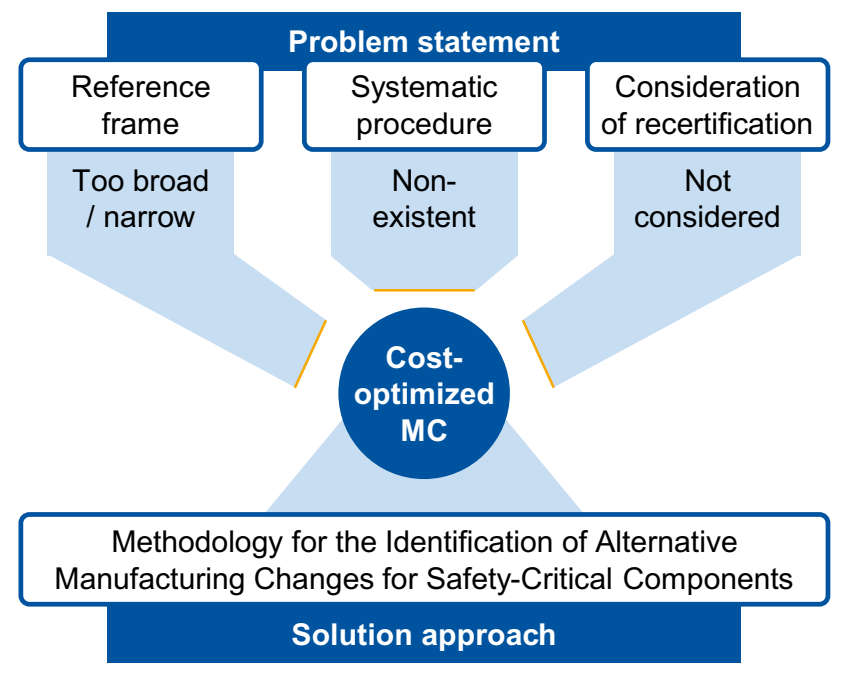

Fig. 1 Problem statement and solution approach 


\section{Methodology}

The initial situation for the methodology's application, its conceptual design and the case study for its validation are introduced in the following section. The steps of the methodology are then presented in detail and applied to the case study. The initial situation, the conceptual design and the case study are depicted in Fig. 2.

The initial situation for the application of the developed methodology arises when a specific component characteristic must be changed. The component is already certified and manufactured via an established and validated process chain. The task at hand is to identify alternative manufacturing changes in the established process chain with which to implement the required change in the component characteristic. The conceptual design of the developed methodology therefore consists of three steps. In the first step, interdependencies within the component's manufacturing process chain are modeled. This model is used in step two to identify process steps which are potentially adaptable to implement the change in the component characteristic. In step three, specific manufacturing changes (e.g. parameter adjustment, tool change, machine substitution) are generated for the identified process step. The steps involved in the methodology are explained in detail in the following subsections, and they are applied to a case study for the purposes of explanation and validation. For the case study, the manufacture of a lower pump housing (component) for a left ventricular assist device (LVAD) is studied. The LVAD is implanted in weakened human hearts to support the blood circulation and extend the waiting time for a donor heart. The validated process chain for the manufacture of LVADs is simplified to processes milling, drilling and drag grinding. The required characteristic change (initial situation) for the case study is a reduction in roughness $R a$ from 0.1 to $0.08 \mu \mathrm{m}$ within the blood channel of the lower pump housing. For reasons of clarity, a limited number of characteristics of the lower pump housing will be considered in the following.

\subsection{Modeling the established manufacturing process chain}

The first step of the methodology involves modeling the interdependencies between the process chain and the manufactured component. The resulting model forms the basis for identifying adaptable process steps. A Multiple Domain Matrix (MDM) according to Eppinger et al. is applied (cf [32]) to create the model. The MDM consists of rows and columns for every characteristic of the considered component as well as for every process step within the established process chain. Characteristics are assigned to features, which are defined as information carriers that refer to geometric or other attributes of the component and can be used to plan the development or production of a component (e.g. blood channel, joining surface) [33]. Process steps are different manufacturing tasks carried out in a single manufacturing process (e.g. roughing and finishing during milling) [34]. A binary representation of interdependencies is used within the MDM to account for the required detail of information in the methodology's next steps and the increasing effort involved in acquiring more detailed but not prerequisite information. In addition, a binary representation makes it

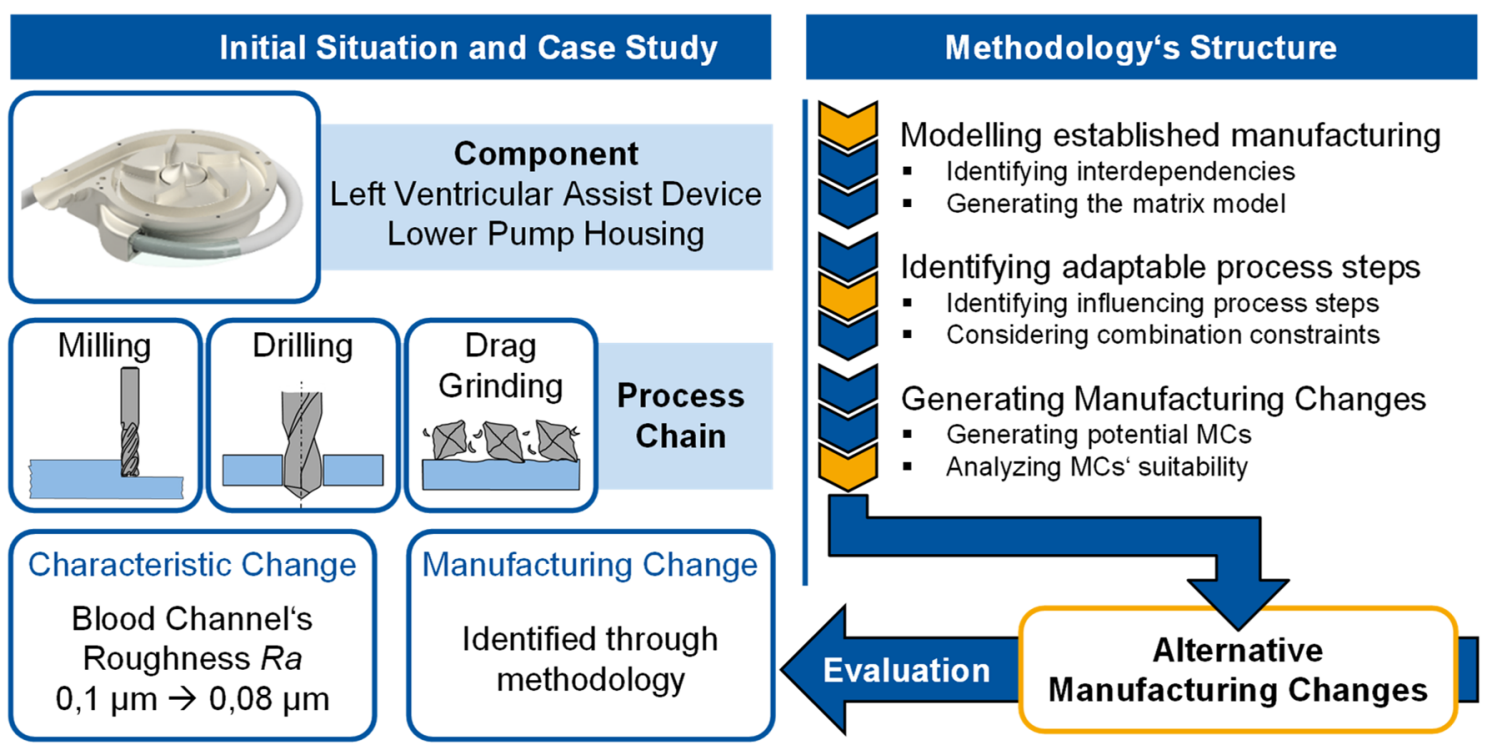

Fig. 2 Initial situation, case study and conceptual design of the methodology 
possible to process the matrix on a computer, which will improve the methodology's industrial applicability in future research. The application of MDMs to model interdependencies within manufacturing was first presented by Hoang et al., who used an MDM to model interdependencies between a process, resources and a product for single mechatronic systems [35]. A simplified MDM for the LVAD is depicted in Fig. 3 and will be used as a reference for further explanations. Since the process chain to be modeled is already established, information about interdependencies are potentially known or can be collected by analyzing existing data such as information about characteristic values from a quality management system or measurement protocols. In addition, information can be acquired from expert interviews or a literature search. Since the information about existing interdependencies forms the basis for the further application of the methodology, this step must be performed in a considered manner. If the information contains uncertainties (e.g. expert interviews), approaches for handling information uncertainties such as [36] can be used.

After the MDM was set up, interdependencies between characteristics $(\mathrm{C} \rightarrow \mathrm{C})$ are identified in the upper left area of the MDM (cf. Fig. 3 (1)). C $\rightarrow$ C interdependencies exist, if characteristics are related to one another and the value of one characteristic may change depending on the other. An example of such interdependencies is the relationship between surface roughness and hardness. If surface roughness increases, roughness peaks are less resistant to plastic deformation and lower hardness values are measured [37]. If a change in the row's characteristic results in a change of the column's characteristic, a " 1 " is assigned to the MDM's cell connecting the characteristics. Although $\mathrm{C} \rightarrow \mathrm{C}$ interdependencies are not relevant for the identification of MCs, they are considered in order to produce a holistic model, which is needed for the analysis of change propagation in later steps following the identification of MCs described in this article. The second type of interdependencies to be modeled in the MDM's upper right area exist between characteristics and process steps (C $\rightarrow$ P, cf. Fig. 3 (2)). $\mathrm{C} \rightarrow \mathrm{P}$ interdependencies describe the relationship between the input and output characteristics of a process step. If the process step remains unaltered and a change in the value of an input characteristic leads to a change in the characteristic's output value, a $\mathrm{C} \rightarrow \mathrm{P}$ interdependency exists between the characteristic and the process step. In this case, a " 1 " is assigned to the cell connecting the characteristic (row) and the process step (column). The interdependency between the blood channel's surface roughness and the drag grinding process serves as an example, since a higher input surface roughness results in a higher output surface roughness after the drag grinding process, if it remains unaltered. The third type of interdependency is modeled in the MDM's lower left

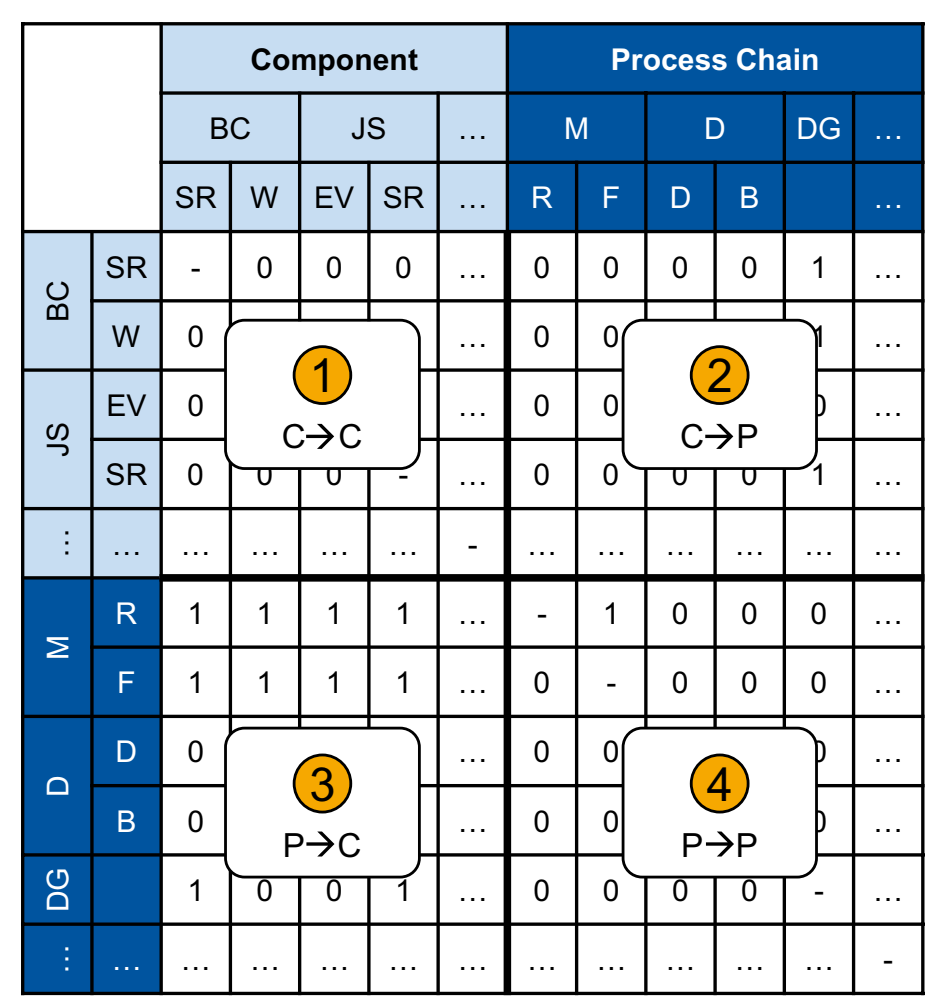

\begin{tabular}{l}
\hline Legend \\
\hline BC: Blood Channel \\
JS: Joining Surface \\
SR: Surface Roughness \\
W: Width \\
F: Flatness \\
EV: Evenness \\
M: Milling \\
D: Drilling \\
DG: Drag Grinding \\
R: Roughing \\
F: Finishing \\
B: Boring \\
\hline 1: Row influencing Column \\
0: No interdependencies
\end{tabular}

Fig. 3 Multiple Domain Matrix model of the established manufacturing 
area and describes the influence of process steps on characteristics ( $\mathrm{P} \rightarrow \mathrm{C}$, cf. Fig. 3 (3)). $\mathrm{P} \rightarrow \mathrm{C}$ interdependencies are the most common ones and exist if a process step influences a characteristic during the manufacturing process, e.g. roughing, finishing and drag grinding influence the blood channel's surface roughness. An existing $\mathrm{P} \rightarrow \mathrm{C}$ interdependency is modeled by assigning a "1" to the corresponding cell. The last type of interdependencies exists between process steps and is modeled in the MDM's lower right area (P $\rightarrow$ P, cf. Fig. 3 (4)). P $\rightarrow$ P interdependencies describe a relationship between two process steps that force both steps to be adapted, although the intention was to only adapt one. An exemplary $\mathrm{P} \rightarrow \mathrm{P}$ interdependency exists between the drilling and the boring process steps, since a change in drilling (e.g. tool with greater diameter) would lead to a forced change of the boring process in order to keep its process condition (depth of cut, cutting force,...) unaltered. An existing $\mathrm{P} \rightarrow \mathrm{P}$ interdependency between two process steps is indicated by a " 1 " in the corresponding MDM's cell. $\mathrm{P} \rightarrow \mathrm{P}$ interdependencies are particularly important in the manufacture of safety-critical components, since a change in process conditions requires coordination with legal agencies to maintain validation, even though no characteristic is influenced by the changed process conditions. Therefore, $\mathrm{P} \rightarrow \mathrm{P}$ interdependencies cannot be defined as combinations of $\mathrm{P} \rightarrow \mathrm{C}$ and $\mathrm{C} \rightarrow \mathrm{P}$ interdependencies, since a combination would only show an existing interdependency if a change in a component characteristic is present, regardless of a change in process conditions.

Having modeled the four types of interdependencies using the MDM, the model is used to identify adaptable process steps. Meanwhile, the MDM's advantage over alternative approaches for modeling manufacturing interdependencies (cf [38]) comes into effect. This advantage lies in the structured and computable processing of manufacturing interdependencies, which makes it possible to automatically identify adaptable process steps.

\subsection{Identifying adaptable process steps}

The second step of the methodology deals with the automatic identification of adaptable process steps with which to implement the required change in a component characteristic (decreasing the blood channel's surface roughness). Therefore, the MDM model is combined with an algorithmic procedure, which processes the MDM model's rows, columns and cells to identify adaptable process steps. The procedure is separated into four sub-procedures. First, the process steps generally influencing the considered characteristic are identified. This set of process steps is then reduced by excluding the process steps that do not influence the characteristic's final value after the last process of the process chain. In the third sub-procedure, the

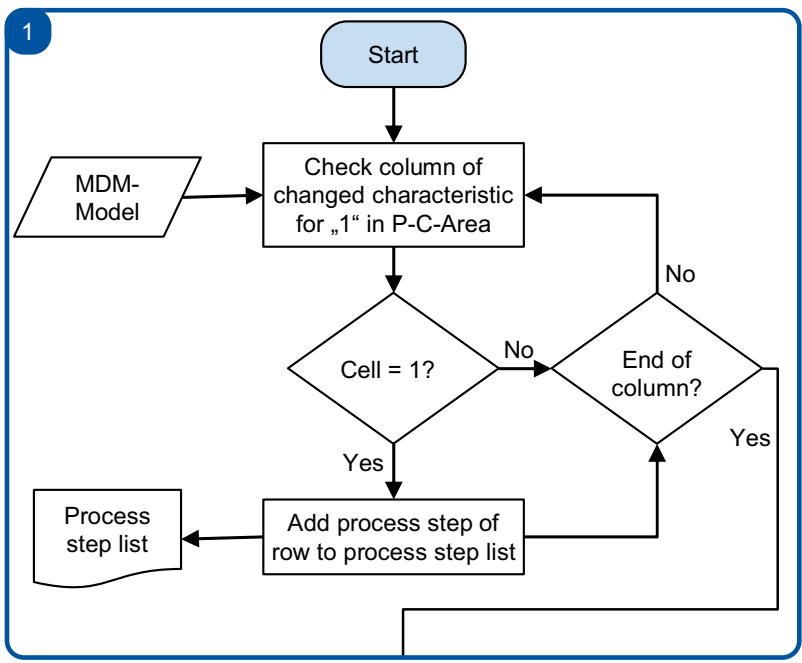

Fig. 4 Procedure to identify process steps influencing the considered characteristic

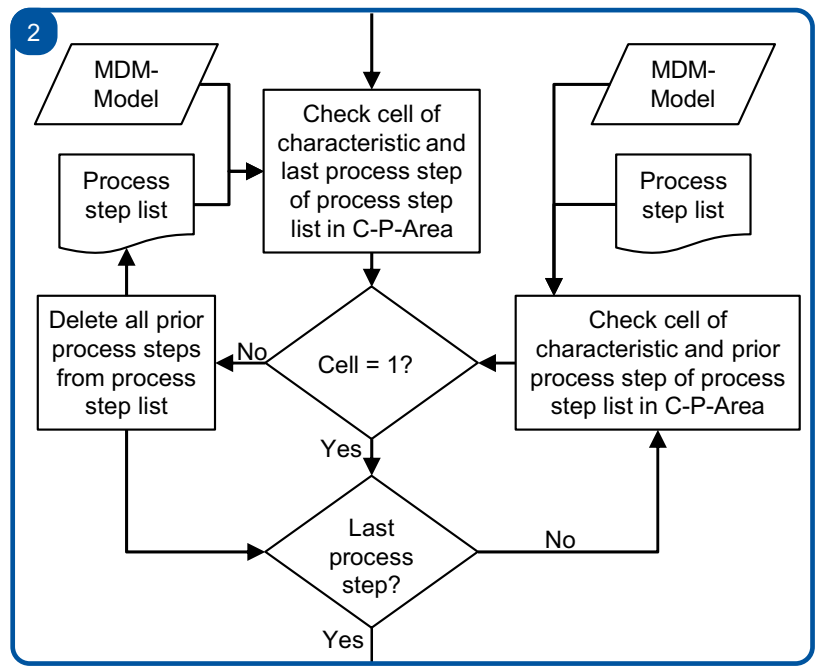

Fig. 5 Procedure to identify process steps influencing the characteristic's final state

remaining process steps are analyzed for combination constraints. Finally, the identified process steps are checked for their ability to change the considered characteristic to the required extent. Since the procedure for the automated identification of adaptable process steps consists of a variety of if-else statements and is supposed to be implemented in a software program, it is depicted in Figs. 4, 5, 6, 7 using flowcharts according to ISO5807 (cf [39]). Due to the high individuality of the methodology's application cases, it is not possible to automatically identify the adaptable process steps in a generic methodology without further input of practitioners. This challenge is taken into account by including user prompts in the flowcharts, 


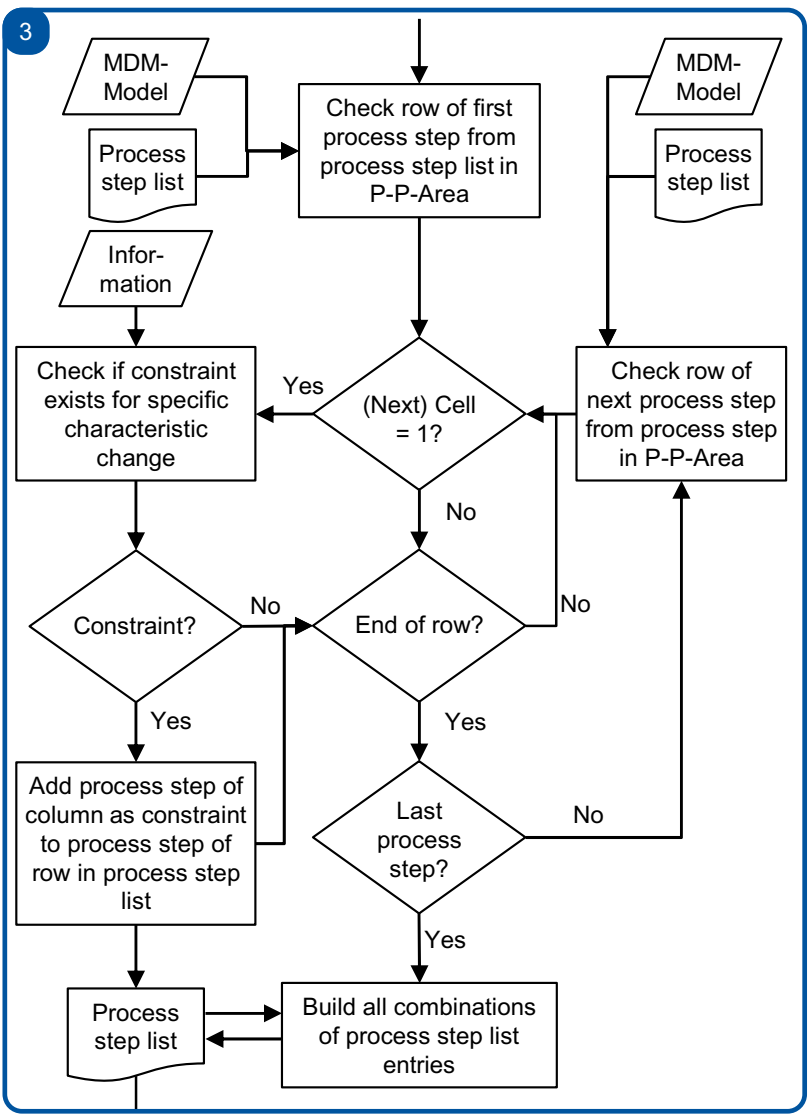

Fig. 6 Procedure to analyze the identified process steps' combination constraints

allowing for the consideration of application specific circumstances.

In the first sub-procedure (cf. Fig. 4), the column of the changed characteristic is scanned for " 1 "s in the MDM's $\mathrm{P} \rightarrow \mathrm{C}$ area (cf. Fig. 3) in order to identify process steps that influence the characteristic during manufacture. These process steps are added to a list (process step list (PSL)) in a sequenced manner. For the case study and the given MDM, the process steps roughing (milling), finishing (milling) and drag grinding were identified to influence the surface roughness of the blood channel.

The second sub-procedure (cf. Fig. 5) reduces the process steps from the PSL to the ones capable of influencing the characteristic's final state. This is done by considering the $\mathrm{C} \rightarrow \mathrm{P}$ interdependencies. If a characteristic prior to a process step in the PSL has no influence on the characteristic after the process step, all process steps of the PSL prior to this process step have no influence on the characteristic's final value at the end of the process chain. This only applies for process steps generally influencing the characteristic (listed in the PSL). Therefore, the process steps in the PSL are analyzed from bottom to top for $\mathrm{C} \rightarrow \mathrm{P}$ interdependencies. For example, if the value of the considered

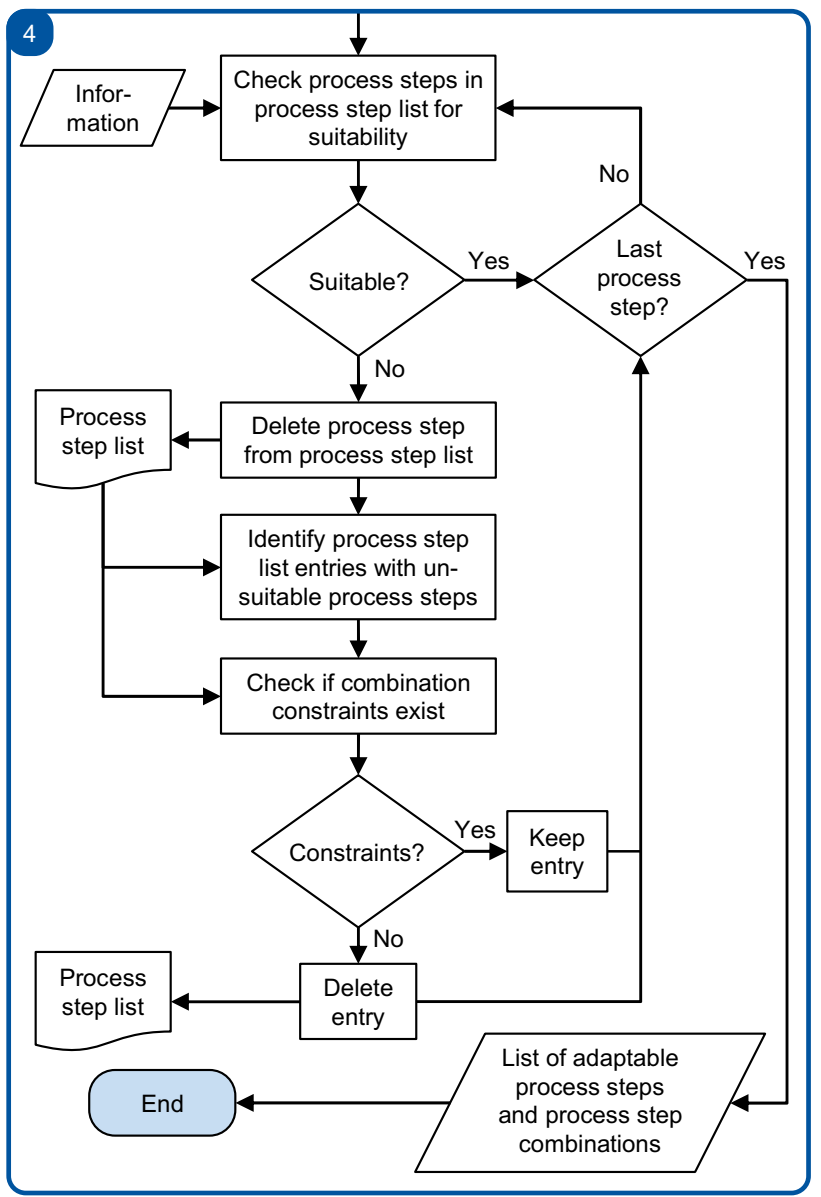

Fig. 7 Procedure to analyze the identified process steps' extent of influence

characteristic before the last process step influencing the characteristic has no influence on the value of the characteristic after the last process step, only this last process step of the process chain has the capability to influence the characteristic's final state. Since there is a $\mathrm{C} \rightarrow \mathrm{P}$ interdependency between the blood channel's surface roughness and the drag grinding process step (cf. Fig. 3), the finishing process step prior to drag grinding is capable of influencing the final value of the surface roughness. In contrast, there is no $\mathrm{C} \rightarrow \mathrm{P}$ interdependency between the surface roughness and the finishing process step, revealing that any modification to the roughing process step will not influence the surface roughness's final value. Therefore, roughing can be deleted from the process step list.

In the third sub-procedure (cf. Fig. 6), the remaining process steps within the PSL are analyzed for combination constraints, forcing the unintended modification of an additional process step. For this, the rows of the process steps are scanned for " 1 "s in the $\mathrm{P} \rightarrow \mathrm{P}$ area of the MDM. The rows of the finishing and drag grinding process steps do not contain any " 1 "s, so there are no constraints. 
For the last sub-procedure (cf. Fig. 7), the MDM is no longer needed. The remaining process steps of the PSL are analyzed for their ability to change the considered characteristic to the required extent, which must be performed by process experts on a case-by-case basis. For example, the final value of a diameter of a shaft may be influenced by a polishing process step and this process step is therefore entered in the PSL. However, the polishing process step may not be capable of influencing the diameter to the required extent (a reduction of $5 \mathrm{~mm}$ ). Therefore, all entries in the process step list must be analyzed with regard to their ability to implement the required characteristic change. Since this ability is highly individual for every case to which the methodology is applied, it cannot be automated by integrating information into the MDM model and must be answered by the technology planners applying the procedure to their specific case. For the case study presented here, the finishing and the drag grinding process steps are capable of achieving the required reduction in surface roughness and therefore remain in the PSL. Finally, all combinations of the remaining process steps are listed, since these combinations also represent applicable adaptations for implementing the required characteristic change. The combination of finishing and drag grinding is added to the PSL, now containing the entries finishing (1), drag grinding (2) and finishing + drag grinding (3).

In summary, the application of the developed procedure to the MDM model results in a list of all adaptable process steps within the established process chain with which to implement the required change in the component characteristic. Although the result of the procedure's application may seem obvious for the simplified exemplary process chain, process chains for the manufacture of safety-critical components are usually highly complex and extensive, creating a need for such a systematic procedure. In order to generate executable manufacturing changes, adaptations to the identified process steps are specified in the next step of the methodology.

\subsection{Generating manufacturing changes}

The last step of the methodology deals with the generation of manufacturing changes by assigning process unspecific adaptations to the previously identified process steps. Therefore, a model comprising and classifying process unspecific adaptations to the manufacture was derived from the scientific literature in the research fields Technology Planning [40], Reconfigurable Manufacturing Systems [41] and Production Systems Engineering [42]. The model of process unspecific manufacturing adaptations is depicted in Fig. 8. It is divided into three types of adaptation: Adaptations to the control of a machine tool, such as changing the cutting speed (parameter) or changing the tool path (strategy),

\begin{tabular}{|l|l|l|}
\hline \multicolumn{2}{|c|}{ Manufacturing Adaptation } \\
\hline \hline Control Level & Material Level & Structural Level \\
\hline \hline Parameter Adap. & Tool Adap. & Add Process \\
Strategy Adap. & Supply Adap. & Delete Process \\
& Machine Adap. & Substitute Process \\
& & Sequence Adap. \\
\hline
\end{tabular}

Fig. 8 Model of process unspecific adaptations in manufacture

adaptations to a machine such as changing tools, supplies or machine components as well as adaptions to the structure of the process chain such as adding, deleting or substituting a process. The model supports technology planners by providing a framework from which to select adaptations and by reducing the complexity of the decision-making process by excluding certain levels (control, material or structural levels) from the decision-making. The classification already indicates the required amount of validation the companies have to deal with, increasing from adaptations to the control level to adaptations to the structural level. Changes on the control level may already be part of the technical documentation of the changed process and therefore do not necessarily require a new validation. For changes on the material level, new validations are mandatory but can be reduced to validations for the one process that is changed. Changes on the structural level are mostly classified as major changes and therefore require a full revalidation of the manufacture. Determining the extent of the required revalidation efforts, taking the already existing certification into account, plays a major role. For the manufacture of aerospace components, a Generic Manufacturing Process Validation allows for small changes within an already validated process parameter window, so that revalidation is not necessary [43]. In the field of medical technology, a legal agency determines whether a manufacturing change is classified as a major change requiring full revalidation or as a minor change requiring partial revalidation or only a change in the existing documentation [8]. The following MCs are therefore generated on the basis of the existing certification.

First, company specific boundary conditions are taken into account by excluding adaptation levels or single adaptations from the application to individual or all of the previously identified process steps. For example, if a machine has recently been bought, substituting this machine must be excluded from further considerations for obvious economic reasons. After that, every combination of the adaptions (cf. Fig. 8) and process steps is analyzed for its ability to implement the required change in the component characteristic. For example, the analysis covers whether a parameter adaption or an adaption of supplies to the drag grinding process 
would be able to reach the new required value of the changed characteristic. The analysis of the combinations results in specific alternative manufacturing changes needed to implement the required change in the component characteristic. Since the determination of potential combinations is highly case specific and requires extensive technological knowledge as well as experiments, this step is carried out in close collaboration between experts for the considered process and the technology planners applying this methodology. Combinations of adaptions are also applicable to process steps. The combinations of the process unspecific adaptations from the model (cf. Fig. 8) with the previously identified process steps generate specific manufacturing changes. Due to the high individuality of process chains and components, a generic procedure to derive specific alternative manufacturing changes cannot be provided. However, the model of general adaptions in combination with the previously identified process step supports technology planners in generating specific manufacturing changes.

For the lower housing of the LVAD, four manufacturing changes were found to be suitable to reduce the blood channel's surface roughness:

1. Adding a finishing cycle to the finishing process step (strategy adaptation),

2. Changing the finishing tool and increasing the drag grinding process time (tool adaptation + parameter adaptation),

3. Increasing the drag grinding process time (parameter adaptation),

4. Substituting the drag grinding process via a laser polishing process.

Alternative manufacturing changes for the implementation of the required component change were thereby identified through the systematic application of the methodology's final step. The alternative MCs form the basis for further analyses and evaluation, which are required to holistically plan MCs for safety-critical components. The analysis and evaluation of MCs will be discussed in future publications, building on the results presented here.

\section{Summary and outlook}

In this article, a methodology for the systematic identification of manufacturing changes for safety-critical components was introduced and applied to a medical technology case study. The methodology supports technology planners in identifying adaptations to an established manufacture in order to implement a required change to a component characteristic. The identification is divided into three steps. In the first step, four kinds of interdependencies between the manufactured component and the established process chain are modelled using a Multiple Domain Matrix. On the basis of this model, an algorithmic procedure using the line and row structure of the model is applied in the second step to identify adaptable process steps influencing the final state of the considered characteristic. In the third step, the identified process steps are matched with general adaptations to the manufacture, which are classified in a developed model. By matching the identified process steps with the process unspecific adaptations from the developed model, alternative process specific adaptations with which to implement the required component change, and hence alternative manufacturing changes, are generated.

The limitations of the methodology introduced here are its reliance on information about interdependencies (c.f. Sect. 3.1), which can be difficult to acquire, as well as itscurrently-highly manual execution. The information about interdependencies is crucial for the methodology's subsequent steps and must therefore be handled considerately. More research is required into the acquisition of the information regarding interdependencies utilized in Sect. 3.1. In addition, the validation has so far been carried out for one case study and more practical applications of the methodology are therefore required for a more conclusive validation.

Future research activities will focus on the analysis and evaluation of manufacturing changes. Within the analysis, a systematic procedure for detecting unintended characteristic changes caused by manufacturing changes as well as for detecting the propagation of changes in the process chain will be developed. Only by taking unintended effects and the propagation of changes into account, can manufacturing changes for safety-critical components be planned with a sufficient degree of planning certainty in order to keep the amount of validation work to a minimum. Additionally, future research will focus on the evaluation of alternative manufacturing changes to enable technology planners to select an MC best suited for their specific application case and conditions.

Acknowledgements Open Access funding provided by Projekt DEAL. The authors thank the German Research Foundation DFG for research support in the project BE 2542/63-1 "Kostenoptimierte Planung von Änderungen in der Fertigung sicherheitskritischer Bauteile durch systematisches Manufacturing Change Management”.

Open Access This article is licensed under a Creative Commons Attribution 4.0 International License, which permits use, sharing, adaptation, distribution and reproduction in any medium or format, as long as you give appropriate credit to the original author(s) and the source, provide a link to the Creative Commons licence, and indicate if changes were made. The images or other third party material in this article are included in the article's Creative Commons licence, unless indicated otherwise in a credit line to the material. If material is not included in the article's Creative Commons licence and your intended use is not permitted by statutory regulation or exceeds the permitted use, you will need to obtain permission directly from the copyright holder. To view a copy of this licence, visit http://creativecommons.org/licenses/by/4.0/. 


\section{References}

1. Schuh G, Gartzen T, Basse F et al (2016) Enabling radical innovation through highly iterative product expedition in ramp up and demonstration factories. Procedia CIRP 41:620-625. https://doi. org/10.1016/j.procir.2016.01.014

2. ElMaraghy HA (ed) (2009) Changeable and reconfigurable manufacturing systems. Springer series in advanced manufacturing, Springer, London

3. Olmez H, Hassannezhad M, Ball N et al (2018) Modelling Change within an integrated approach to manufacturing system design. Proc Int Des Conf Des 2018:611-622. https://doi. org/10.21278/idc.2018.0401

4. Koch J (2017) Manufacturing change management-a process based approach for the management of manufacturing changes. Technische Universität München, Diss.

5. International Organization for Standardization (2016) Medical devices-quality management systems-requirements for regulatory purposes (13485)

6. International Organization for Standardization (2015) Aerospace series-quality systems-first article inspection requirements (9102)

7. Regulation (EU) $2017 / 745$ of the European Parliament and of the Council of 5 April 2017 on medical devices, amending Directive 2001/83/EC, Regulation (EC) No 178/2002 and Regulation (EC) No 1223/2009 and repealing Council Directives 90/385/EEC and 93/42/EEC

8. EWG Medical Device Directive (2007) Richtlinie 93/42/EWG DES RATES

9. Hermann L, Rey J, Bergs T (2019) Methodology for an integrative manufacturing change management in technology planning for medical products. Proc Int Conf Compet Manuf 2019:470-476

10. Klocke F, Brockmann M, Gierlings S et al. (2012) Adaptive control of manufacturing processes for a new generation of jet engine components. Proc CIRP Int Conf Intell Comput Manuf Eng (7)

11. Ogrodnik PJ (2020) Medical device design: Innovation from concept to market, 2nd edn. Academic Press, London

12. Harer J, Baumgartner C (eds) (2018) Anforderungen an Medizinprodukte: Praxisleitfaden für Hersteller und Zulieferer, 3, vollständig, überarbeitete edn. Hanser, München

13. Bisdas T, Bohan P, Lescan M et al (2019) Research methodology and practical issues relating to the conduct of a medical device registry. Clin Trials 16(5):490-501. https://doi.org/10.1177/17407 74519855395

14. Klocke F, Fallböhmer M, Kopner A et al (2000) Methods and tools supporting modular process design. Robot Comput Integr Manuf 16(6):411-423. https://doi.org/10.1016/S0736-5845(00)00024-7

15. Regulation (EU) $2017 / 746$ of the European Parliament and of the Council of 5. April 2017 on in vitro diagnostic medical devices and repealing Directive 98/79/EC and Commission Decision 2010/227/EU

16. Eversheim W, Schuh G (eds) (1999) Produktion und Management: Gestaltung von Produktionssystemen. Hütte, Springer, Berlin

17. Klocke F, Müller J, Mattfeld P et al (2016) Integrative technology and inspection planning of medical devices. Procedia CIRP 51:105-110. https://doi.org/10.1016/j.procir.2016.05.097

18. Müller J (2017) Integrative Gestaltung von Fertigungsprozessund Prüffolgen für sicherheitskritische Bauteile. Diss, RWTH Aachen

19. Schindler S (2014) Strategische Planung von Technologieketten für die Produktion. Technische Universität München, Diss.
20. Denkena B, Dittrich M-A, Jacob S (eds) (2019) Methodology for integrative production planning in highly dynamic environments, vol. 13. Springer

21. Jacob A, Windhuber K, Ranke D et al (2018) Planning, evaluation and optimization of product design and manufacturing technology chains for new product and production technologies on the example of additive manufacturing. Procedia CIRP 70:108-113. https://doi.org/10.1016/j.procir.2018.02.049

22. Koch J, Gritsch A, Reinhart G (2016) Process design for the management of changes in manufacturing: toward a manufacturing change management process. CIRP J Manuf Sci Technol 14:10 19. https://doi.org/10.1016/j.cirpj.2016.04.010

23. Malak RC, Aurich JC (2013) Software tool for planning and analyzing engineering changes in manufacturing systems. Procedia CIRP 12:348-353. https://doi.org/10.1016/j.procir.2013.09.060

24. Plehn C (2017) A method for analyzing the impact of changes and their propagation in manufacturing systems. Technische Universität München, Diss.

25. Cichos D, Aurich JC (2017) Integration of a physical model into the realization of engineering changes in manufacturing systems. AMM 869:159-166. https://doi.org/10.4028/www.scientific.net/ AMM.869.159

26. Karl F, Reinhart G (2015) Reconfigurations on manufacturing resources: Identification of needs and planning. Prod Eng Res Devel 9(3):393-404. https://doi.org/10.1007/s11740-015-0607-x

27. Hoang X-L, Fay A, Marks P et al. (2017) Generation and impact analysis of adaptation options for automated manufacturing machines. In: 2017 22nd IEEE International Conference on Emerging Technologies and Factory Automation (ETFA). IEEE, pp 1-8

28. Marks P, Hoang XL, Weyrich M et al (2018) A systematic approach for supporting the adaptation process of discrete manufacturing machines. Res Eng Design 24(3):1-21. https://doi. org/10.1007/s00163-018-0296-5

29. Schulz C, Mannuß O (2015) Sustainability structured process FMEA for pharmaceutical products. In: Proceedings of the 4th annual world conference of the society for industrial and systems engineering, pp 161-167

30. Rathore AS, Winkle H (2009) Quality by design for biopharmaceuticals. Nat Biotechnol 27(1):26-34. https://doi.org/10.1038/ nbt0109-26

31. Martinez-Marquez D, Mirnajafizadeh A, Carty CP et al (2018) Application of quality by design for 3D printed bone prostheses and scaffolds. PLoS ONE 13(4):e0195291. https://doi. org/10.1371/journal.pone.0195291

32. Eppinger SD, Browning TR (2012) Design structure matrix methods and applications. Engineering systems. MIT Press, Cambidge

33. Salomons OW, van Houten FJAM, Kals HJJ (1993) Review of research in feature-based design. J Manuf Syst 12(2):113-132. https://doi.org/10.1016/0278-6125(93)90012-I

34. Grünebaum T, Hermann L, Trauth D et al (2019) Towards sustainable production: a methodology to assess influences between life cycle phases in tool manufacturing. Procedia CIRP 80:376-381. https://doi.org/10.1016/j.procir.2019.01.001

35. Hoang X-L, Marks P, Weyrich M et al (2017) Modeling of interdependencies between products, processes and resources to support the evolution of mechatronic systems. IFAC-PapersOnLine 50(1):4348-4353. https://doi.org/10.1016/j.ifacol.2017.08.873

36. Rey J, Apelt S, Trauth D et al (2019) Highly iterative technology planning: Processing of information uncertainties in the planning of manufacturing technologies. Prod Eng Res Devel 13(3-4):361371. https://doi.org/10.1007/s11740-019-00882-7

37. Kim J-Y, Kang S-K, Lee J-J et al (2007) Influence of surfaceroughness on indentation size effect. Acta Mater 55(10):35553562. https://doi.org/10.1016/j.actamat.2007.02.006 
38. Wuest $\mathrm{T}$ (2015) Identifying product and process state drivers in manufacturing systems using supervised machine learning. Springer, Heidelberg

39. International Organization for Standardization (1985) Information processing - documentation symbols and conventions for data, program and system flowcharts, program network charts and system resources charts (5807)

40. Buchholz S (2014) Bewertung des Substitutionsrisikos von Fertigungssystemen. Diss, RWTH Aachen

41. Azab A, ElMaraghy H, Nyhuis P et al (2013) Mechanics of change: a framework to reconfigure manufacturing systems. CIRP J Manuf Sci Technol 6(2):110-119. https://doi.org/10.1016/j.cirpj .2012 .12 .002
42. Eichgrün K (2003) Prozesssicherheit in fertigungstechnischen Prozessketten: Systemanalyse, ganzheitlich Gestaltung und Führung. Diss, TU Kaiserslautern

43. U.S. Department of Transportation Federal Aviation Administration (2017) Type Validation and Post-type Validation Procedures (8110.52B)

Publisher's Note Springer Nature remains neutral with regard to jurisdictional claims in published maps and institutional affiliations. 